\title{
Chip-Based Capillary Zone Electrophoresis Mass Spectrometry (CZE-MS) for Rapid Resolution and Quantitation of Critical Quality Attributes in Protein Biotherapeutics - Supporting Information
}

\author{
Andrew B. Dykstra* ${ }^{*}$, Tawnya G. Flick ${ }^{\ddagger}$, Burton Lee, Laura E. Blue, Nic Angell.
}

Amgen, Inc., Department of Attribute Sciences, Thousand Oaks, CA 91320, United States.

Supporting Information Figure 1. Evaluation of carryover.

Supporting Information Figure 2. Evaluation of carryover (zoom of Supporting Information Figure 1).

Supporting Information Figure 3. Linearity of peptide family XXXXNXXXX from $0.15 \mu \mathrm{M}$ to $30 \mu \mathrm{M}$.

Supporting Information Figure 4. MSMS corresponding to modified peptide in Figure 5A labeled as "IsoAsp."

Supporting Information Figure 5. MSMS corresponding to modified peptide in Figure 5B labeled as "Deamidation 1."

Supporting Information Figure 6. MSMS corresponding to modified peptide in Figure 5B labeled as "Deamidation 2."

Supporting Information Figure 7. MSMS corresponding to modified peptide in Figure 5C labeled as "Met Oxidation."

Supporting Information Figure 8. MSMS corresponding to modified peptide in Figure 5D labeled as "Trp Oxidation."

Supporting Information Figure 9. Comparison of attribute quantitation between Injection Group 1 (first injection for each condition) and Injection Group 4 (injections for each condition performed after $\sim 10 \mathrm{~h}$ for $\mathrm{mAb}$ and $\sim 14 \mathrm{~h}$ for BiS). 
Supporting Information Figure 1. Evaluation of carryover. Water injections were performed as Injection 1 and Injection 119 on the same HR chip. No additional water cycles were performed between injection 1 and Injection 119. Signal intensity is normalized to total ion electropherogram intensity of a representative sample injection (NL: 1.80E9).

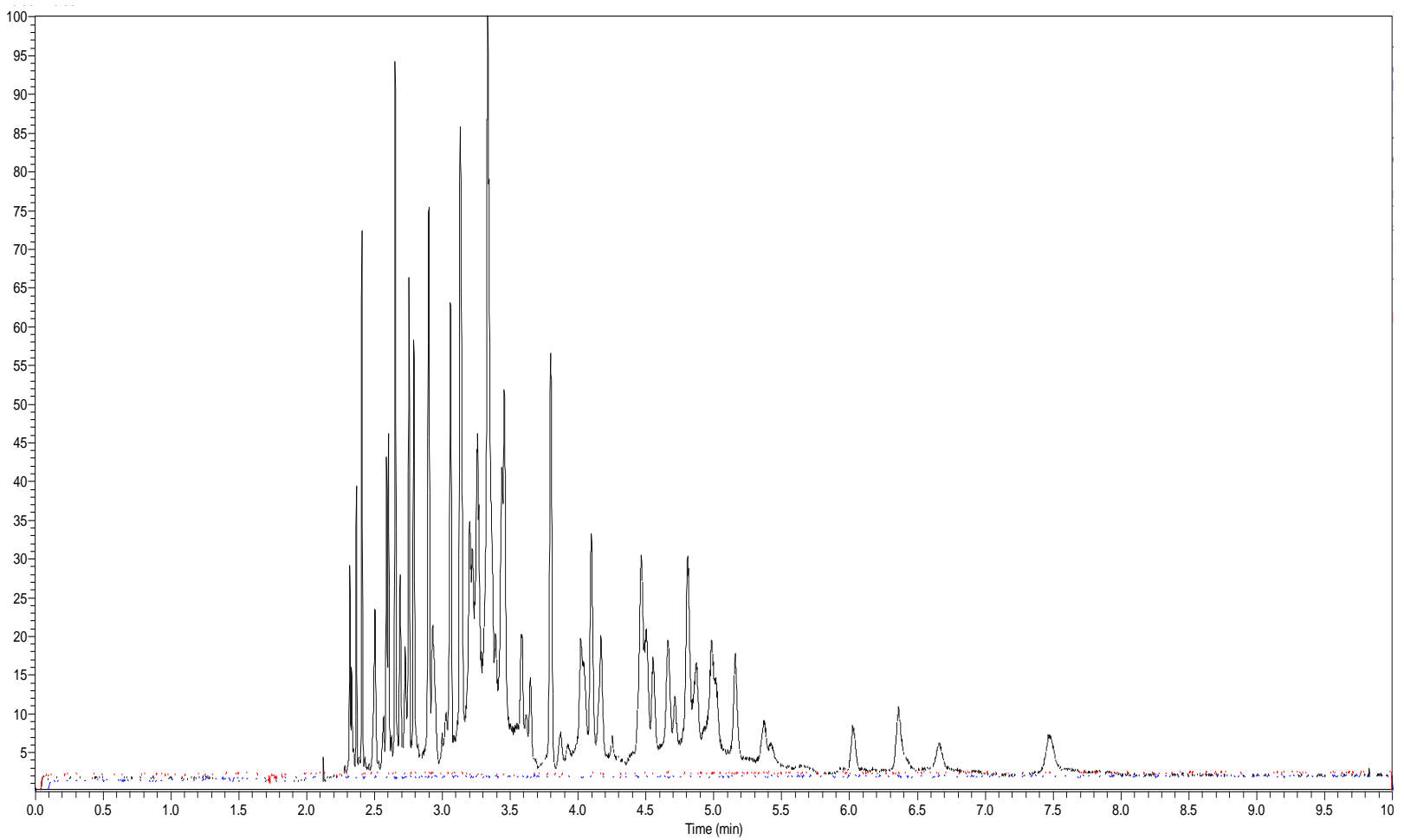


Supporting Information Figure 2. Evaluation of carryover (zoom of Supporting Information Figure 1).

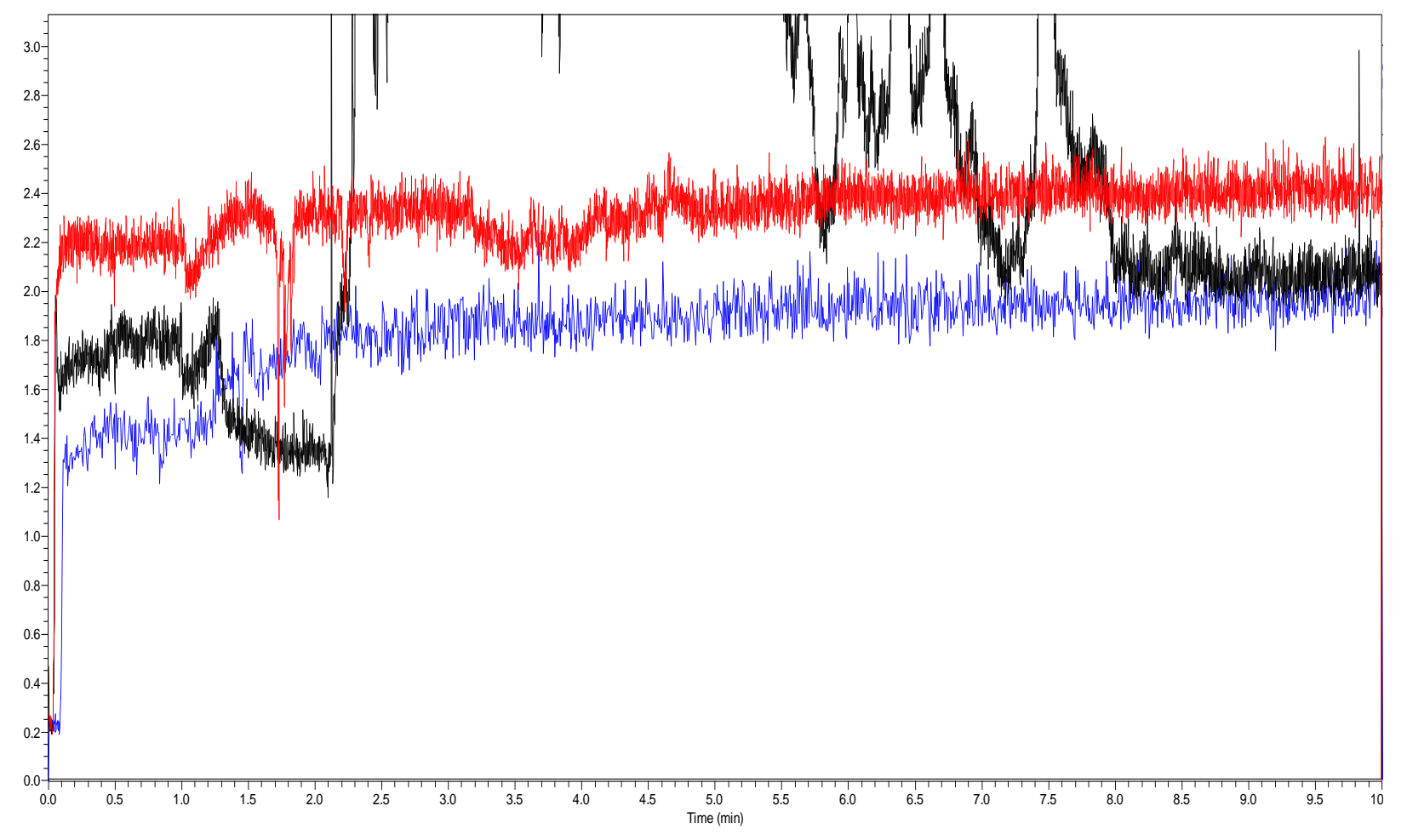


Supporting Information Figure 3. Linearity of peptide family XXXXNXXXX from $0.15 \mu \mathrm{M}$ to $30 \mu \mathrm{M}$.

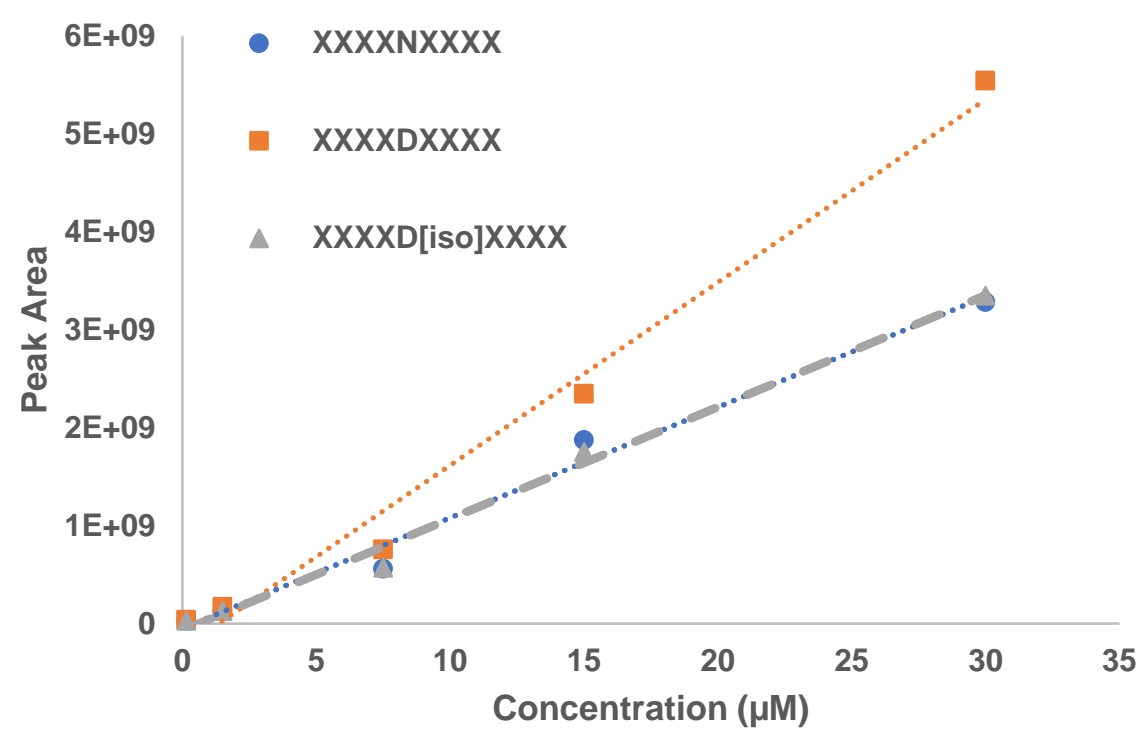


Supporting Information Figure 4. MSMS corresponding to modified peptide in Figure 5A labeled as "IsoAsp." Only b and y ions are annotated.

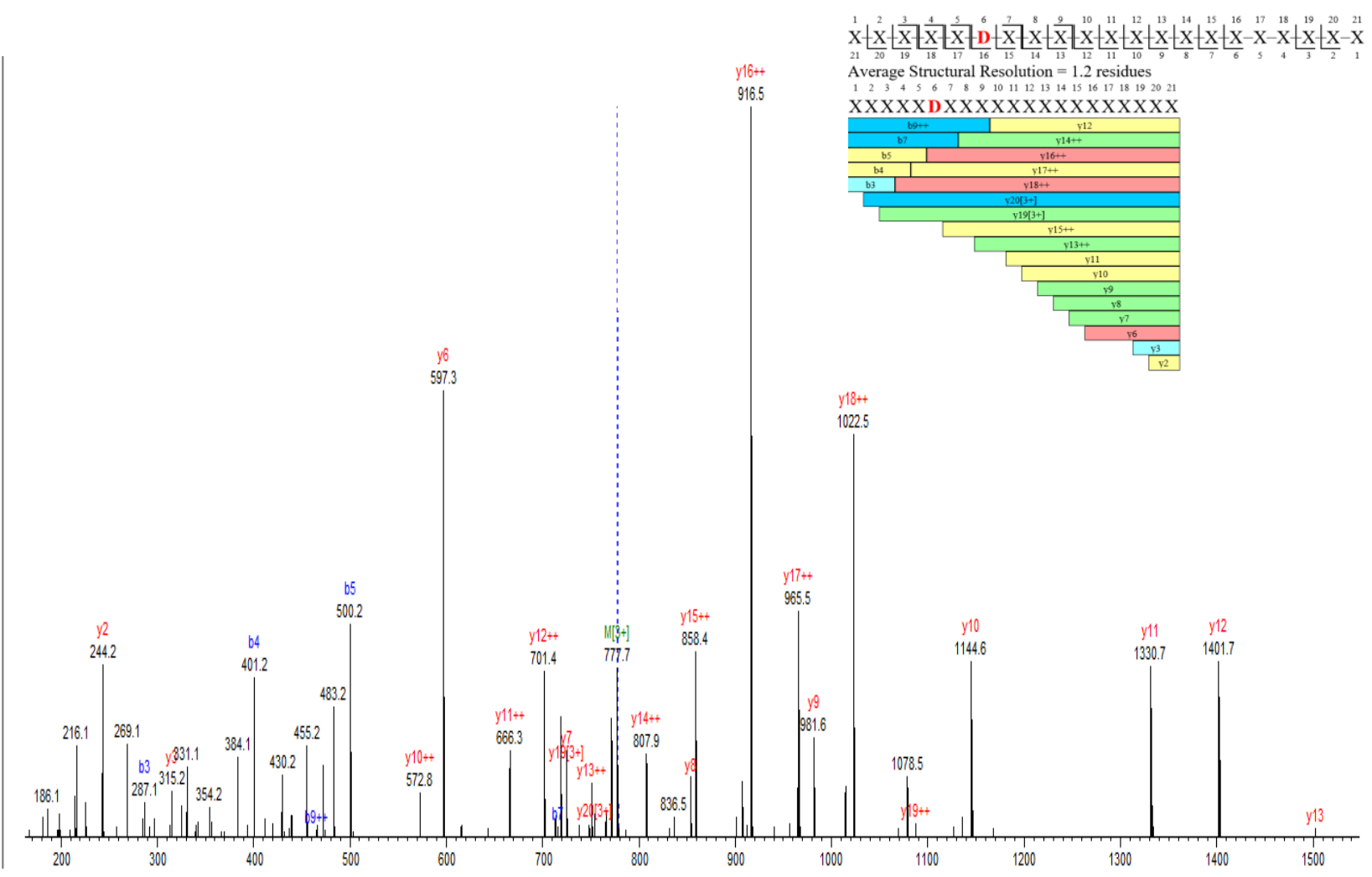


Supporting Information Figure 5. MSMS corresponding to modified peptide in Figure 5B labeled as "Deamidation 1." Only b and y ions are annotated.

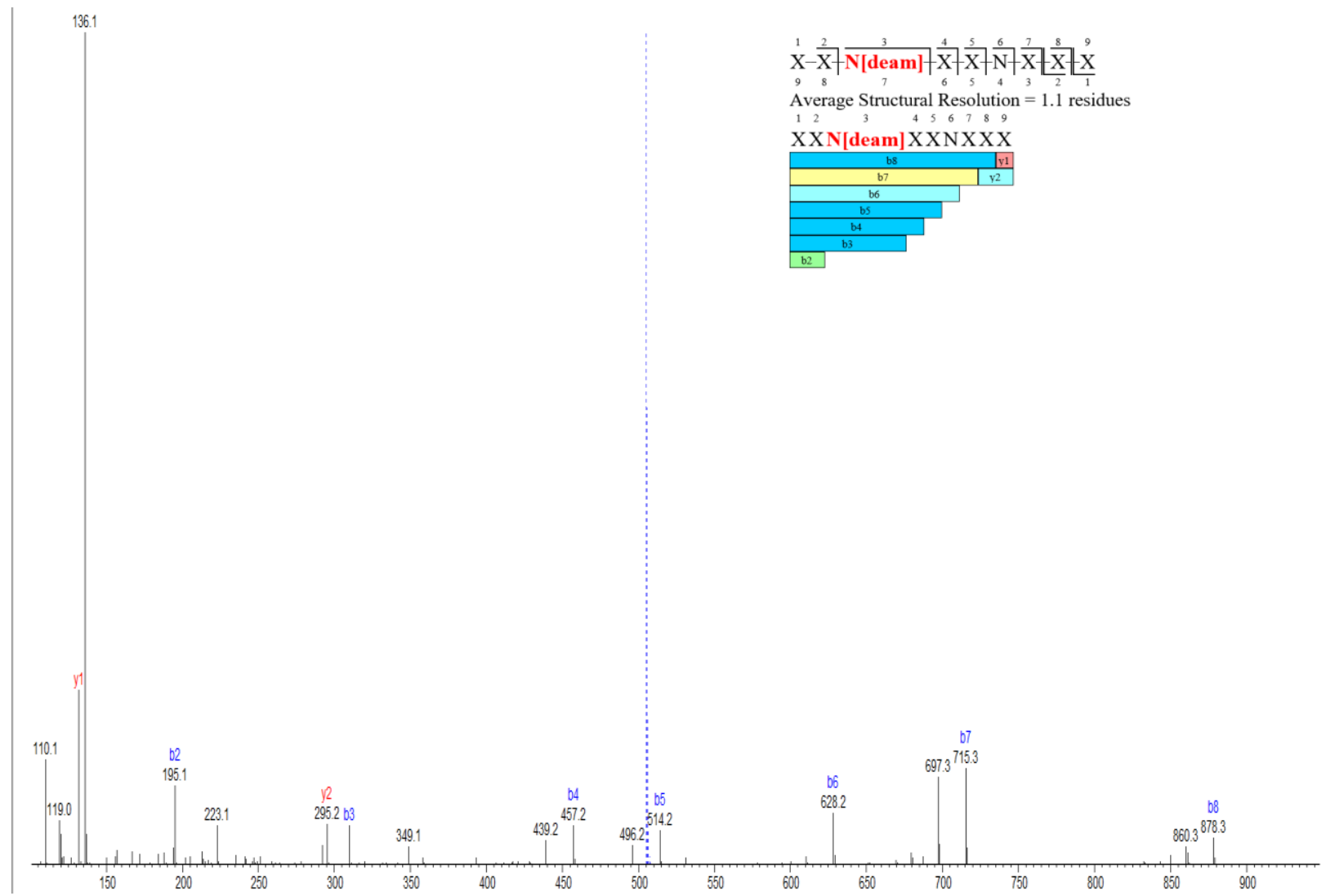


Supporting Information Figure 6. MSMS corresponding to modified peptide in Figure 5B labeled as "Deamidation 2." Only b and y ions are annotated.

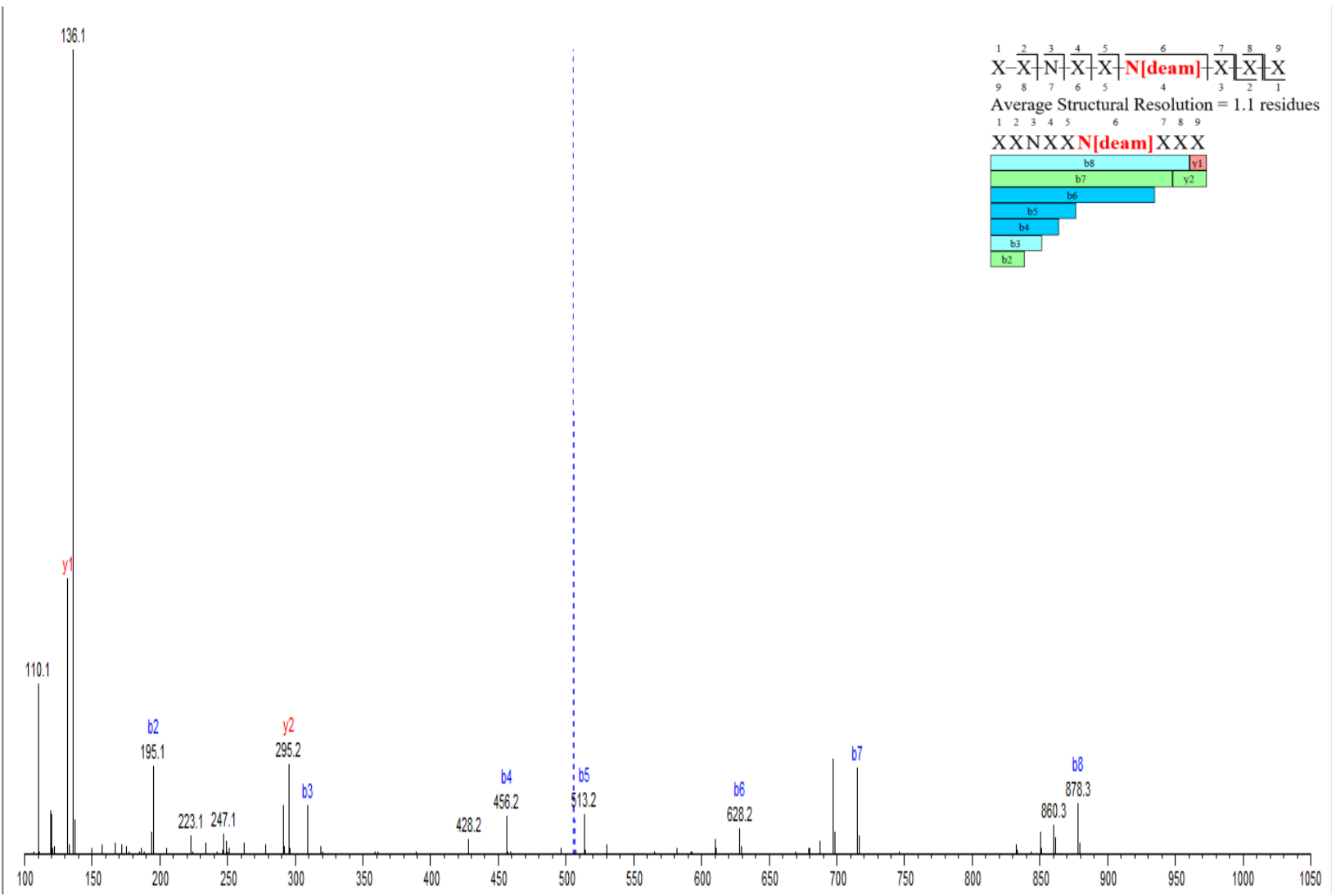


Supporting Information Figure 7. MSMS corresponding to modified peptide in Figure 5C labeled as "Met Oxidation." Only b and y ions are annotated.

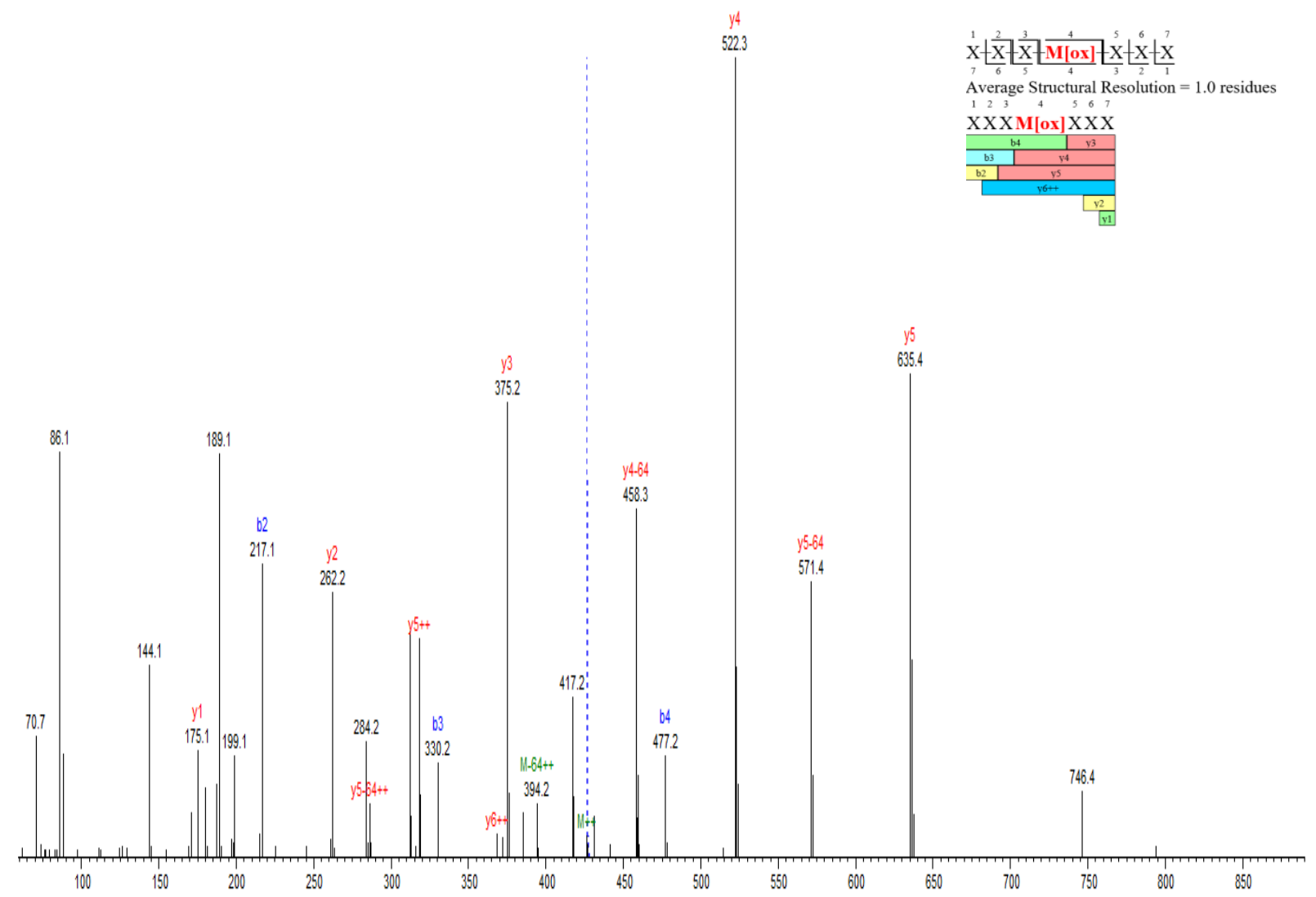


Supporting Information Figure 8. MSMS corresponding to modified peptide in Figure 5D labeled as "Trp Oxidation." Only b and y ions are annotated.

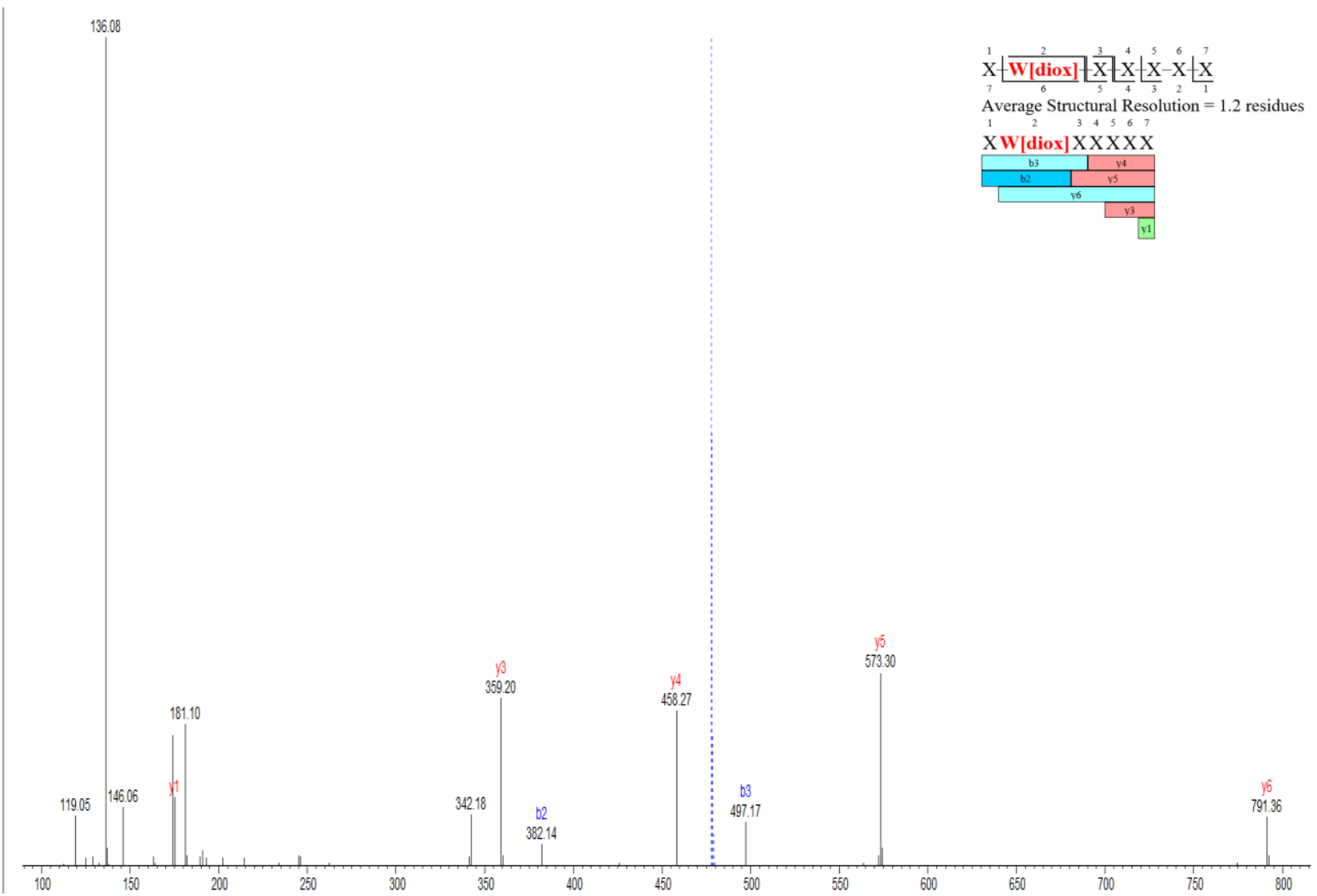


Supporting Information Figure 9. Comparison of attribute quantitation between Injection Group 1 (first injection for each condition) and Injection Group 4 (injections for each condition performed after $\sim 10 \mathrm{~h}$ for $\mathrm{mAb}$ and $\sim 14 \mathrm{~h}$ for $\mathrm{BiS}$ ).

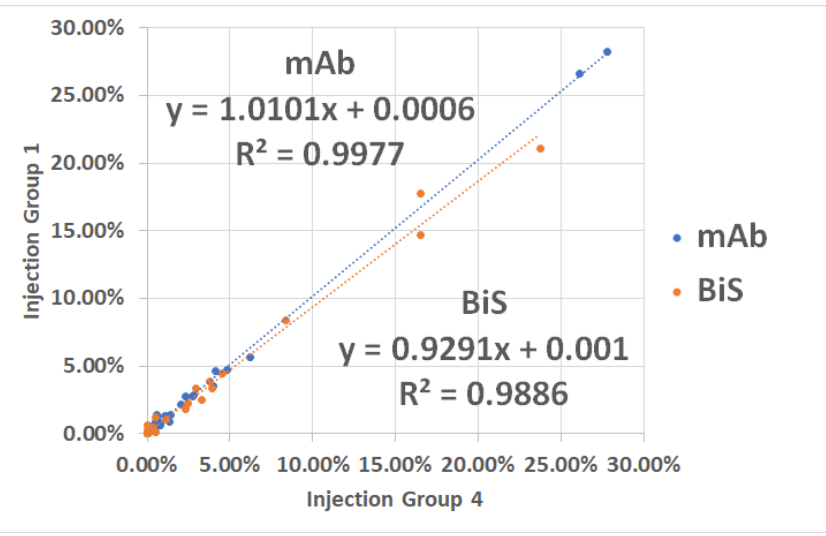

